\title{
Abrasive waterjet intensifier model for machine diagnostics
}

\author{
Gianni Ferretti $^{1} \quad$ Michele Monno $^{2} 3$ Bruno Scaglioni ${ }^{13}$ Massimo Goletti $^{2} 3$ Marco Grasso ${ }^{2} 3$ \\ ${ }^{1}$ Dipartimento di Elettronica, Informazione e Bioingegneria DEIB, Politecnico Di Milano, \\ Via Ponzio 34/5, 20133 Milano, Italy \\ ${ }^{2}$ Dipartimento Meccanica, Politecnico di Milano, via La Masa 1, 20156, Milano, Italy \\ ${ }^{3}$ MUSP Lab, Via Tirotti 9, Le Mose, 29122 Piacenza, Italy
}

\begin{abstract}
This paper investigates the dynamics of a waterjet plant with multiple phased single-acting plungers. An object oriented dynamic model is proposed and discussed. The simulator may be tuned to generate signals under different health conditions to train multi-fault diagnosis tools. In fact, due to the challenging pressure conditions and the aggressiveness of abrasive materials, the reliability of machine tool components is a major concern. The information throughput provided by the model is validated with respect to real-industrial data, acquired in reference cutting scenarios.

Keywords: Waterjet Cutting; High Pressure Pump; Object-Oriented Modeling; Condition Monitoring
\end{abstract}

\section{Introduction}

Waterjet/abrasive waterjet (AWJ) cutting machines are used for several industrial applications thanks to the great flexibility of the technology, which is suitable for cutting a wide range of materials [Kovacevic et al., 1997]. This kind of machine tool includes an Ultra High Pressure (UHP) pump to generate the necessary pressure energy that is then converted into kinetic energy by the orifice into the cutting head. Different components, either belonging to the UHP pump or to the cutting head, are subject to different kinds of faults and performance degradation, due to the challenging pressure conditions and the aggressiveness of abrasive particles.

The reliability of AWJ cutting machines is therefore a topic of major concern in industry. A fast detection of a faulty state and the automatic identification of the root cause for observed symptoms are expected to provide several benefits, including the reduction of unexpected machine stops, a quick leakage recovery, the minimization of wastes, the enhancement of maintenance operations, etc. There are several studies in the literature devoted to AWJ process monitoring [Peržel et al., 2012, Krenickỳ and Rimár, 2012, Axinte and Kong, 2009, Rabani et al., 2012, Choi and Choi, 1997], mainly related to the determination and possible improvement of the cut quality. Nevertheless, very few authors investigated the development of automated tools for in-process monitoring and diagnosis of machine tool health conditions [Annoni et al., 2009, Grasso et al., 2013, Grasso et al., 2014]. One of major challenges consists of characterizing the AWJ plant behavior under both healthy and faulty conditions, in order to train fault classifiers.

Real data under faulty states are always difficult and expensive to collect, which makes purely data-driven diagnostic methods poorly attractive for a practical use. Model-based methods are expected to yield more effective diagnostics capabilities, thanks to the possibility of simulating the plant behavior under different operating conditions.

This paper investigates the dynamics of an AWJ plant with multiple phased single-acting plungers and it represents a first attempt to design an objectoriented dynamic model for such a kind of system. The model may be tuned to generate simulated signal patterns under different health conditions in order to train multi-fault diagnosis tool. Moreover, the model's behavior can be compared with the measurements and give indications on the failures, a diagnostic method based on object-oriented models is proposed in [Bunus and Lunde, 2008]. The proposed model generates simulated water pressure and plunger displacement patterns, which can be used to characterize the AWJ working cycle. The injections of degraded states and faulty conditions into the model allows to characterize the pattern deviations from the natural state, and hence to develop novel model-based fault detection and classification toolkits. The real industrial data include signals under healthy states and in the presence of faults affecting either the UHP pump components (cracked cylin- 
ders) or the cutting head components (broken orifices). The paper is organized as follows: Section 2 describes the model, in Section 3 a comparison between experimental results and simulations is presented in case of normal and faulty conditions, and the model tuning is presented. Section 4 concludes the paper.

\section{UHP intensifier model}

In this section, the model of the CMS Tecnocut $60 \mathrm{HP}$ intensifying machine is described, a picture of the device is reported in Fig. 1. This intensifier is able to reach more than 4000 bars by means of 3 single-acting cylinders with a maximum water flow rate of $5 \mathrm{l} / \mathrm{min}$ (orifices up to $0.4 \mathrm{~mm}$ in diameter). The machine scheme is reported in Fig. 2.

The intensifier is based on the Pascal principle: an oil circuit is pressurized by means of an electric pump, the oil flows into a single acting cylinder with a ratio of areas $1 / v$ producing a pressure in the water circuit given by the input oil pressure multiplied by $v$. It must be pointed out that at least three cylinders are required in order to keep a quasi-constant output pressure, since the work cycle of the machine is composed by three strokes: the pre-compression stroke, where the outlet valve of the cylinder is closed and the oil flowing into the chamber heightens the water pressure, the compression stroke, where the outlet valve is open and the water flows from the cylinder to the orifice, and finally the back stroke, where the piston returns to the original position. The oil circuit is composed of two subcircuits, the high-pressure oil circuit depicted in black, and the back-stroke circuit depicted in red, the oil is pressurized by a variable displacement piston pump equipped with an hydraulic feedback control system.

It must be pointed out that the timing mechanism of the plungers is controlled by a PLC based on contactless proximity sensors. This mechanism, along with the displacement control system of the electric pump, plays a crucial role in the water pressure signal behavior. As will be seen later, the modeling phase of these components has been carried out in great detail.

The water circuit (blue in Fig. 2), is composed by a series of high pressure pipes connecting the outlet ports of the cylinders to the final orifice. The Modelica model of the intensifier has been carried out my means of the Hydraulics library [Hyd, 2014], which provided all the basic components for the model construction. In particular, fluid components for compressible water and oil are unavoidable in the application of interest, where the extremely high pressures compresses water by a factor

\section{of $20 \%$.}

The fluids properties are made available to the hydraulic components by means of the inner/outer statements, hence the global machine model has been split in two main components, namely the water and oil circuits, where two different fluids have been adopted. The submodels are connected by means of translational 1D flanges, which represent the cylinders interfaces.

The water circuit, shown in Fig. 3, is composed by three chambers representing the part of the cylinders in contact with water, the supply circuit and the final orifice, modeled as an hydraulic resistance. It must be pointed out that the length and compliance of the pipe connecting the pressure intensifier and the final orifice are not negligible, hence a longline component has been used. The purpose of the aforementioned component is to model the dynamics of long pipes, such as the water hammer effect, and to consider the compliance of the pipe's walls subject to the water pressure. The final orifice was modeled by means of the Orifice model, a component of the Hydraulics library, where either laminar or turbulent flow can be used depending on the pressure drop on the component. The thermal effects on the orifice were not considered as the machine is self-coolant and no significant temperature gradient was detected, nor reported in literature.

The model of the oil circuit, shown in Fig. 4 is more involved, as it contains the oil pistons, the PLC, the group of mechanisms that feeds the oil to the cylinders and the variable displacement pump with its hydraulic feedback control system.

The oil feeding mechanism of the pistons is shown in Fig. 5. The circuit's valves are directly controlled by the PLC but the commutation delays are not symmetric with respect to the command signal, hence the delay is acquired as an external parameter, depending on the direction of commutation. It must be pointed out that valves commutation timing has great consequences on the pressure signal, therefore, accurate tuning of the delays is required. The influence of these parameters will be discussed in Section 3.

The model of the main oil circuit, including the pump and the displacement control system is shown in Fig. 6. The operating nominal pressure of the circuit, which directly controls the water pressure, is regulated by means of a relief valve that opens if the pressure exceeds the nominal value. The pump is equipped with an internal volume regulator, with the aim of optimizing the overall efficiency of the machine with respect to the various operation points. The regulator controls the volume of the pump by applying a pressure on the swash plate. The 
volume is determined by the outflow rate through a system of valves and springs whereof parameters are not known. The dynamics of the control system was modeled as follows:

$$
\begin{gathered}
C_{t}(t)= \begin{cases}1 & \text { if } Q_{\text {out }}<Q_{s} \\
1-\frac{Q_{\text {out }}(t)-Q_{s}}{Q_{m}-Q_{s}} & \text { if } Q_{s}<Q_{\text {out }}<Q_{m} \\
0 & \text { if } Q_{\text {out }}>Q_{m}\end{cases} \\
C(s)=\frac{1}{(1+T s)} e^{-\tau s} C_{t}(s)
\end{gathered}
$$

with $C(s)$ and $C_{t}(s)$ being the Laplace transforms of the control output $C(t)$ and the signal $C_{t}(t)$, while $Q_{s}$ is the flow rate condition of maximum displacement and maximum operative pressure, $Q_{m}$ is the flow rate at which the displacement of the pump is zero and finally $T$ and $\tau$ are the parameters describing the inertia of the system. The parameters of the control system, as well as the valves' delays, were collected from the datasheets [Par, 2011].

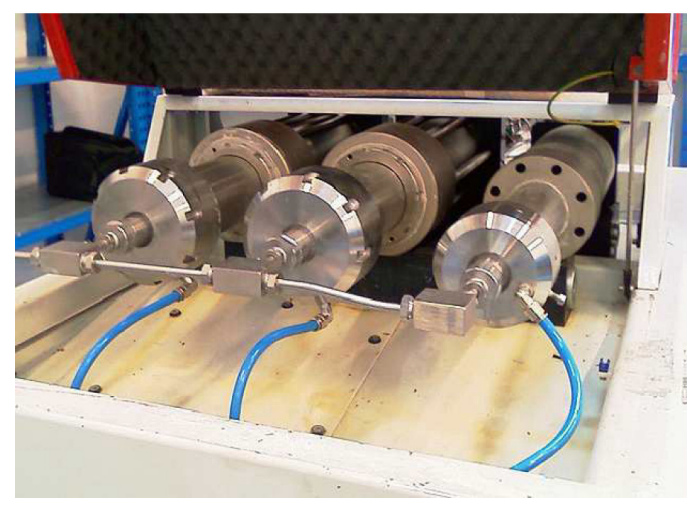

Figure 1: Pressure Intensifier

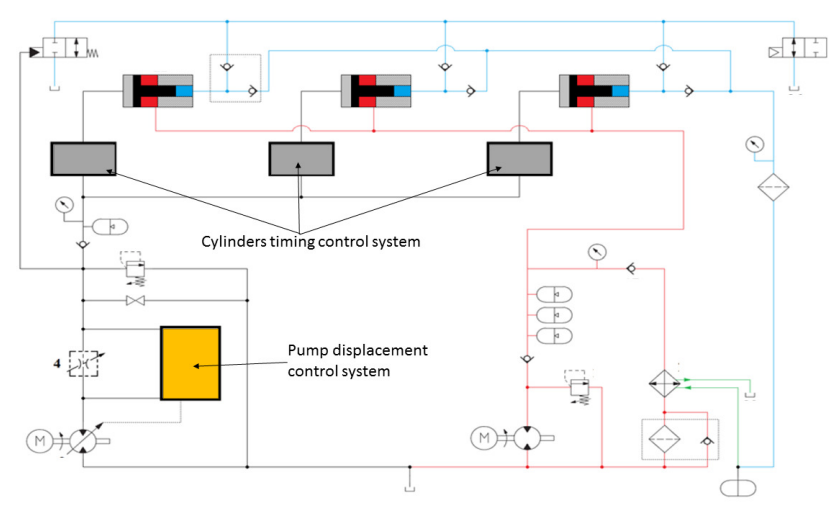

Figure 2: CMS Tecnocut UHP intensifier

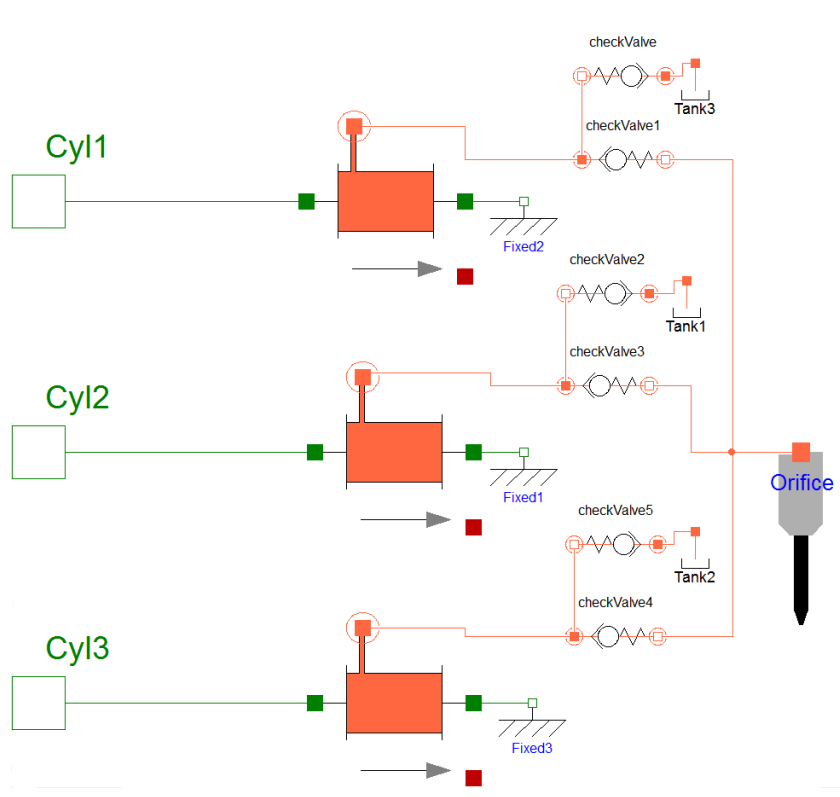

Figure 3: Water Circuit

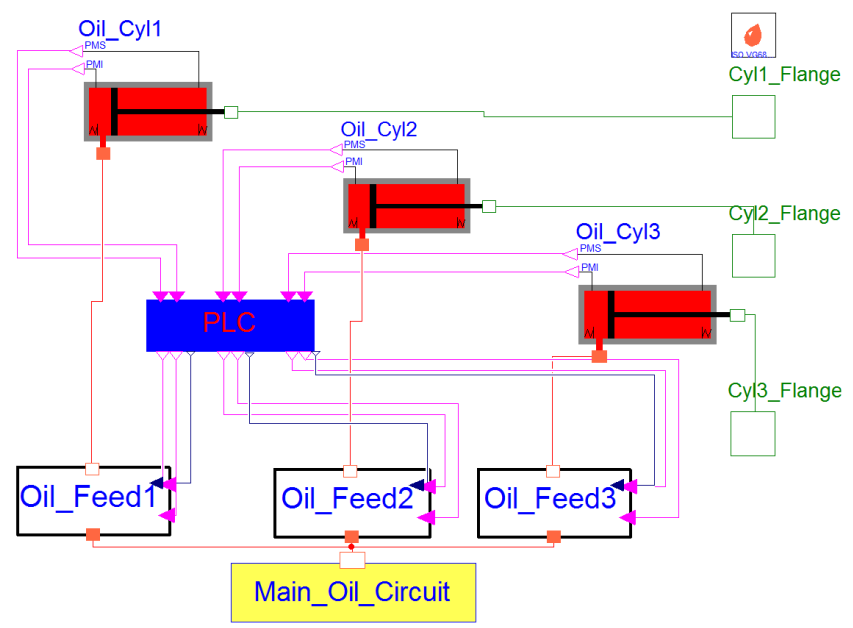

Figure 4: Oil model

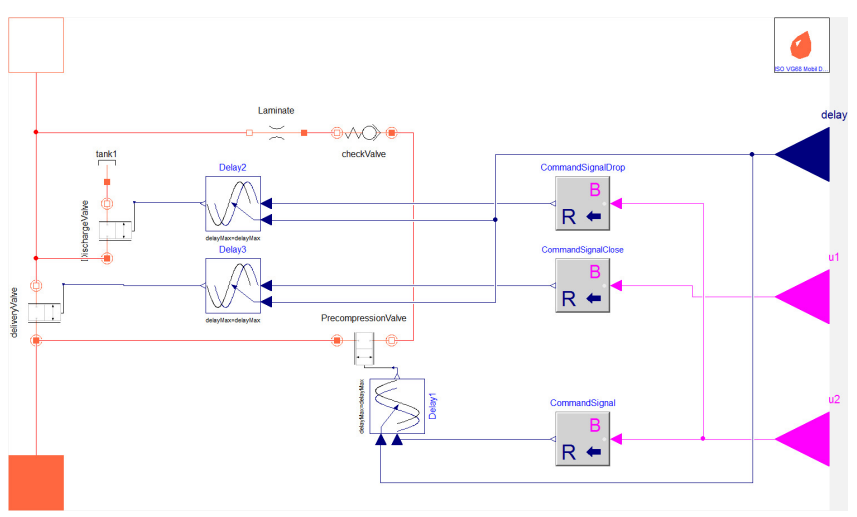

Figure 5: Feed circuit 


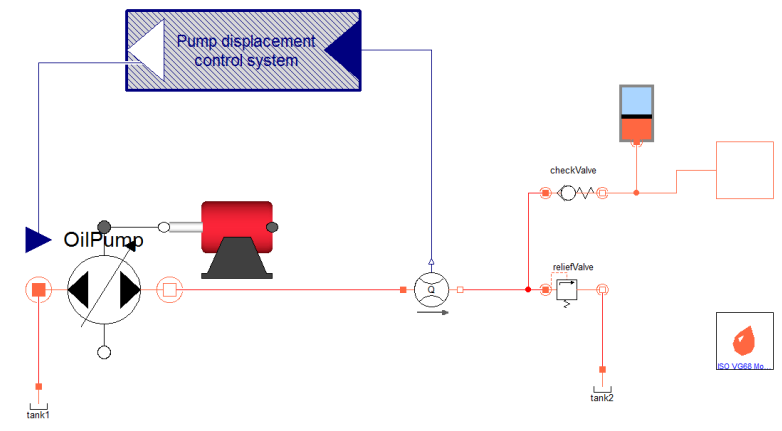

Figure 6: Main oil circuit

\section{Simulation and model tuning}

The model was simulated in the Dymola environment [Dynasim $A B$,$] and validated with respect to experi-$ mental data. The experiments were carried out with a nominal water pressure of 3600 bar and a final orifice of $0.33 \mathrm{~mm}$. The stroke of the three cylinders and the water pressure signal were acquired by means of a pressure sensor and three linear position transducers. It must be pointed out that the simulation of an hydraulic system characterized by pressures in the order of $10^{7}$ $\mathrm{Pa}$ and flow rates of $10^{-6} \mathrm{~m}^{3} / \mathrm{s}$ can run into serious numerical stiffness problems. Moreover the commutation of the hydraulic valves can lead to situations where the pressure drop is close to zero in components with turbulent flow models, leading to numerical problems as the gain $d Q / d P$ of the components goes to infinity as the pressure drop goes to zero, as described in [Hyd, 2014], hence the Esdirk45a integration algorithm has been used with a relative tolerance of $10^{-3}$, which is a higher-order, A-stable algorithm suitable for stiff problems. It must also be pointed out that the classical DASSL algorithm often looses stability with tolerances between $10^{-3}$ and $10^{-5}$, and could even not converge in reasonable time with smaller tolerances.

Fig. 7 shows the comparison between the experimental signal, depicted in red, and the simulated pressure signal, in blue. As it is apparent, the simulation is in good accordance with the experiments, the average pressure is similar as well as the in-cycle fluctuations, nonetheless experimental signals exhibit a decreasing trend with the periodicity of one cycle.

The observation is confirmed by the analysis of the power spectrum of the signals, visible in Fig. 8. A $1 X$ cycle component is visible in the experimental signal but it is missing in the simulated signal. The discrepancy was attributed to a difference between the cylinders' friction coefficients, whose parameter's values are

\begin{tabular}{cc}
\hline Index & Value \\
\hline Simulated signal: Standard deviation & $62.56 \mathrm{bar}$ \\
Experimental signal: Standard deviation & $42.86 \mathrm{bar}$ \\
Correlation Coefficient & 0.7147 \\
\hline
\end{tabular}

Table 1: Model validation results

difficult to obtain. It must be pointed out that the Hydraulics lib implements a friction model described in [Tustin, 1947].

In order to fit the experimental data, the model was updated by assigning different values of viscous damping to the three cylinders. Figures 9 and 10 show the signal and the power spectrum with different damping values, the experimental pressure is faithfully reproduced and the power spectrum correctly identifies the $1 X$-cycle component. The standard deviation and correlation of experimental and simulated pressure signals were compared for the sake of model validation, and the results are shown in Table 3. Note in particular that the correlation coefficient between the signals is higher then 0.7 .

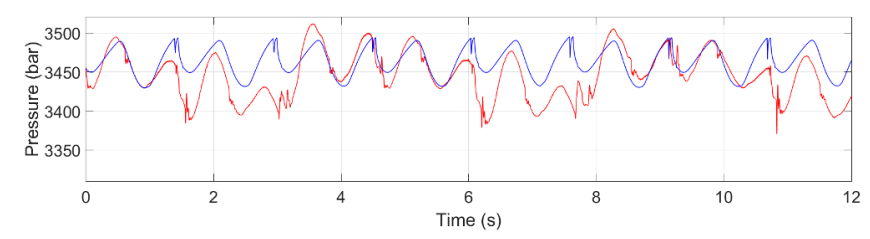

Figure 7: Real signal vs Simulated

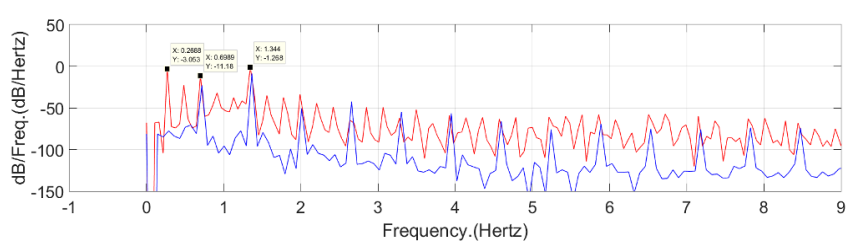

Figure 8: Power spectrum of experimental and simulated pressures

\subsection{Faults injection}

One of the main purposes of the developed model is to act as a virtual test bench for fault simulation, hence, a set of faults whose experimental data were available have been implemented in the model. Then, suitable indicators have been identified, based on the simulated and experimental signals.

One of the most frequent and important fault in the AWJ machines is a crack in the cylinder body, often 


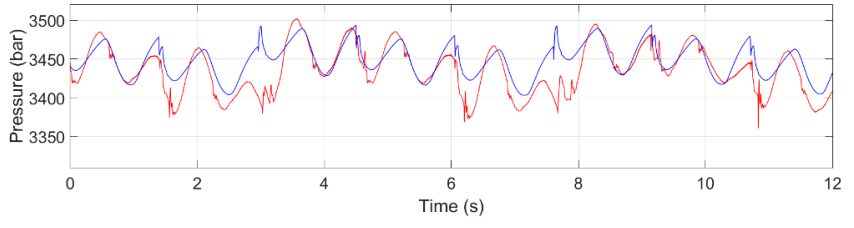

Figure 9: Experimental and simulated pressures with different friction coefficients

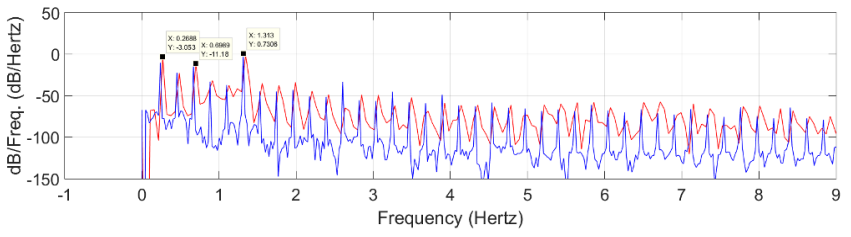

Figure 10: Power spectrum of experimental and simulated pressures with different friction coefficients

caused by the high pressures involved in the process. The fault was modeled as a leakage between the water chamber and the external environment and the conductance parameter was tuned on the advice of the cylinder manufacturer. It must be pointed out that the specific value of the leakage was tuned on the specimen under study, but the qualitative behavior of the system in fault condition is similar for a wide range of the conductance parameter. Fig.11 shows the cepstrum of the pressure signals. The Cepstrum of a signal [Childers et al., 1977] is defined as follows:

$$
X(T)=|\boldsymbol{F}[\ln (|\boldsymbol{F}[x(t)]|)]|
$$

Where $x(t)$ is the signal in the time domain, $X(T)$ is the cepstrum and $\boldsymbol{F}$ is the Fourier transform operator. It represents the Fourier transform of the logarithm of the Fourier transform of the signal, and it is a good indicator of the main harmonics of the signal. In particular it is useful in the analysis of the water pressure as it clearly shows the length of the pumping cycle. The indipendent variable of a cepstrum analysis is the quefrency, measured in seconds. Note that the period of the pumping cycle decreases from 4.8 to 4.2 seconds in both cases, showing a good indicator for this kind of fault. Moreover, the strokes of the cylinders, shown in Fig. 12 exhibit a remarkable difference in the precompression stroke of the cracked cylinder with respect to the remaining ones.

The fault condition caused by a consumed final orifice was also investigated. The physical fault consists in a wear of the orifice, whose diameter increases and assumes irregular shape, with negative effect on the cutting quality. The fault was reproduced experimentally by installing a fatigued orifice on the machine, while in the simulation environment, the diameter of the orifice was set to $0.35 \mathrm{~mm}$ according to the results of a geometrical analysis of the component. In the case of faulty orifice, a global reduction of the pumping cycle duration can be expected rather then a difference between the cylinders stroke signals. Fig. 13 shows the cepstrum of the simulated and experimental signal, the $1 X$ - cycle component of the cepstrum reduces from a quefrency of $4.8 \mathrm{~s}$ to $3.2 \mathrm{~s}$ in the simulation and $3.3 \mathrm{~s}$ in the experiments, showing good accordance.

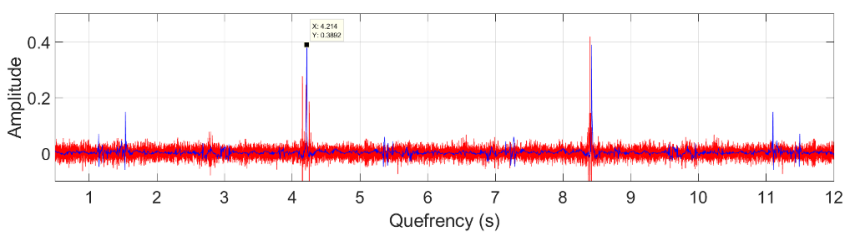

Figure 11: Cepstrum of the pressure signal, cracked cylinder

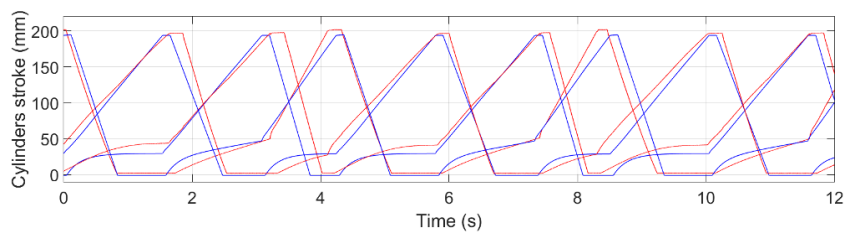

Figure 12: Cylinder strokes in case of fault

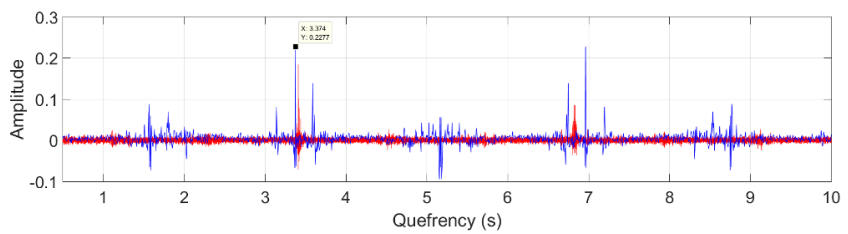

Figure 13: Cepstrum of the pressure signal, broken orifice

\section{Conclusion}

The object-oriented model of a complex machine tool, involving ultra high pressure water circuit, interaction between fluid mechanics and control system was presented. The model was tuned, and subsequently validated by means of a comparison with experiments exerted on a real industrial machine tool. Two different types of fault were introduced with the aim of reproducing the behavior of the machine in the case of malfunction. The developed model lays the groundwork for a model-based condition monitoring system which is expected to yield more effective diagnostics capabilities 
by means of the on-line identification of faulty conditions. The development of the monitoring system will be addressed in a future work.

\section{References}

[Par, 2011] (2011). Parker Hannifin Corporation, Series DIVW, D Style catalog, USA: Parker.

[Hyd, 2014] (2014). Modelon AB - Hydraulics Library - Version 4.1.

[Annoni et al., 2009] Annoni, M., Cristaldi, L., Lazzaroni, M., and Ferrari, S. (2009). Nozzles classification in a high-pressure water jet system. Instrumentation and Measurement, IEEE Transactions on, 58(10):3739-3745.

[Axinte and Kong, 2009] Axinte, D. and Kong, M. (2009). An integrated monitoring method to supervise waterjet machining. CIRP AnnalsManufacturing Technology, 58(1):303-306.

[Bunus and Lunde, 2008] Bunus, P. and Lunde, K. (2008). Supporting model-based diagnostics with equation-based object oriented languages. pages 121-130.

[Childers et al., 1977] Childers, D. G., Skinner, D. P., and Kemerait, R. C. (1977). Cepstrum: A guide to processing. Proceedings of the IEEE, 65(10):14281443.

[Choi and Choi, 1997] Choi, G. S. and Choi, G. H. (1997). Process analysis and monitoring in abrasive water jet machining of alumina ceramics. International Journal of Machine Tools and Manufacture, 37(3):295-307.

[Dynasim AB, ] Dynasim AB. Dymola. Lund, Sweden.

[Grasso et al., 2013] Grasso, M., Goletti, M., Annoni, M., and Colosimo, B. M. (2013). A new approach for online health assessment of abrasive waterjet cutting systems. International Journal of Abrasive Technology, 6(2):158-181.

[Grasso et al., 2014] Grasso, M., Pennacchi, P., and Colosimo, B. (2014). Empirical mode decomposition of pressure signal for health condition monitoring in waterjet cutting. The International Journal of Advanced Manufacturing Technology, 72(1-4):347364.
[Kovacevic et al., 1997] Kovacevic, R., Hashish, M., Mohan, R., Ramulu, M., Kim, T., and Geskin, E. (1997). State of the art of research and development in abrasive waterjet machining. Journal of manufacturing science and engineering, 119(4B):776-785.

[Krenickỳ and Rimár, 2012] Krenickỳ, T. and Rimár, M. (2012). Monitoring of vibrations in the technology of awj. In Key Engineering Materials, volume 496, pages 229-234. Trans Tech Publ.

[Peržel et al., 2012] Peržel, V., Hreha, P., Hloch, S., Tozan, H., and Valíček, J. (2012). Vibration emission as a potential source of information for abrasive waterjet quality process control. The International Journal of Advanced Manufacturing Technology, 61(1-4):285-294.

[Rabani et al., 2012] Rabani, A., Marinescu, I., and Axinte, D. (2012). Acoustic emission energy transfer rate: a method for monitoring abrasive waterjet milling. International Journal of Machine Tools and Manufacture, 61:80-89.

[Tustin, 1947] Tustin, A. (1947). The effects of backlash and of speed-dependent friction on the stability of closed-cycle control systems. Electrical Engineers - Part IIA: Automatic Regulators and Servo Mechanisms, Journal of the Institution of, 94(1):143-151. 